\title{
ENGINEERING INSTRUCTOR USE AND INTERPRETATION OF RUBRICS IN A LARGE SCALE UNDERGRADUATE INDEPENDENT RESEARCH COURSE
}

\author{
Nikita Dawe, Lisa Romkey and Alan Chong \\ University of Toronto \\ nikita.dawe@mail.utoronto.ca; lisa.romkey@utoronto.ca; alan.chong@utoronto.ca
}

\begin{abstract}
This work extends prior research into the efficacy and validity of rubrics designed for a large scale fourth year capstone course at the University of Toronto. In the context of this required course - in which projects span the gamut from industry-partnered design projects to research oriented theses - rubrics serve several important functions for students, supervisors and administrators. In this paper, we describe a set of interviews conducted with project supervisors (faculty) and the key findings based on these interviews. A number of interesting themes emerged, on the perceptions and use of the rubrics, views on the purpose of the rubric, and on specifics of rubric design. The results of this work will support the development of a survey and, eventually, changes to rubric design and deployment.
\end{abstract}

Keywords: Assessment, Rubric, Capstone Project

\section{INTRODUCTION}

Creating common rubrics for multidisciplinary capstone courses poses a significant challenge, considering the diversity of projects pursued by engineering students in their final year. The specific course under study is the fourth year Engineering Science thesis course, which is a required course for all Engineering Science students at the University of Toronto. In previous papers, we have described the challenges posed, given the variety and number of projects included in the course. The Engineering Science program includes 8 diverse Majors, and students are permitted to work with any Faculty member at the University of Toronto on their thesis, and so the thesis projects engage with a number of different disciplinary and academic traditions. Each year, the thesis course supports $170-200$ projects, supervised by well over 100 professors from $25+$ academic departments.

Common rubrics serve in a number of capacities for the key stakeholders involved in the course. For students, they function as detailed assignment guides, setting expectations for their various deliverables in the course. For supervisors, the rubrics also establish a common set of expectations across multiple performance levels, helping to facilitate efficient feedback. For administrators, they provide key data for graduate attributes collection (across multiple attributes) and help contribute to some degree of uniformity of experience between supervisors and projects.

In previous papers, we have described the challenges posed to universal rubric development by the variety and number of projects, as well as the number of supervisors, who have varying levels of experience managing an undergraduate capstone experience [1-3, 8]. Attempts to validate the rubrics on the student side - through focus groups and interviews - have led to important insights into how they understand and employ the rubrics [8], revealing an anxiety about how supervisors use them (or don't use them) as assessment tools. These concerns lead us to test for inter-rater reliability, comparing the results of two "rubric expert" raters with the supervisors: we identified substantial agreement between the "rubric experts," but only fair agreement between those raters and actual project supervisors [3]. This paper probes that question further, and describes the results of a qualitative study in which project supervisors participated in semi-structured interviews about their experience using the rubrics to better understand their perspectives. The final report rubric, along with the "rubric guide", that was the main focus of the study can be found in Appendix A.

\section{INTERVIEW METHODOLOGY}

An interview protocol was developed based on previous research investigating how assessors use and interpret universal rubrics $[4,6]$. The interview protocol first posed demographic and experience questions, including questions about the supervisors' experience using rubrics (in the thesis course or otherwise). After this was completed, the interviews highlighted four main themes: (1) Rubric use: how and when they use the rubric, whether they use the rubric guide, and whether they consider the rubric to be useful; (2) Rubric content: the inclusion of appropriate or irrelevant criteria, whether the rubric contradicted any requirements or expectations, and ordering of criteria; (3) Rubric clarity: overall clarity, and specific questions about criteria that have been identified in the past as problematic; and (4) Guidance and training 
materials: use of the rough guide, and exploring the possibility of other training materials and opportunities.

Eighteen thesis supervisors (Faculty members from the University of Toronto) were selected for interviews, based on a purposeful sampling strategy [7] to ensure that a variety of disciplines, project orientations and experience levels were represented in the population under study. In total, 11 supervisors agreed to be interviewed.

Each interview took approximately 30-45 minutes, and all of the interviews were recorded and transcribed. As noted, the interviews focused primarily on the final rubric used at the end of the course to assess the major report and overall project experience. The interviewer followed the interview protocol; however, she also allowed for some flexibility within the interviews, so that participants could emphasize themes of interest, allowing for candid conversation which resulted in richer content. Follow up questions were asked to clarify or to redirect supervisors when they interpreted questions differently than expected. The interviewer took brief notes but relied on recordings rather than notetaking to preserve the flow of discussion. Recordings were transcribed after all interviews were completed.

\section{DATA ANALYSIS}

After the interviews were transcribed, two members of the research team engaged in a data coding process. First, one member of the research team used an inductive, opencoding process, described by Grbich [5], involving line-byline analysis to identify a set of specific concepts and categories. Initially, content from the interviews deemed relevant to the research study was highlighted. Then, this content was organized into a preliminary list of categories. Next, three transcripts were reviewed in detail, and each section of text (sometimes by sentence, sometimes by word or short phrase) was coded to the existing list of categories, and additional categories were added when needed.

Then, a second member of the research team utilized a variation of memo writing, also described by Grbich [5]. This provided an opportunity to respond to content and categories, develop new insights and create some connections between the themes in the data. After this process was finished, the categories and notes were reviewed again, and a final list of three major themes, with a number of sub-categories, was created.

\section{RESULTS AND DISCUSSION}

The data analysis resulted in a number of interesting themes, which have been organized under three major categories: (1) Perceptions of the rubric, given the nature (or perceived nature) of the thesis course; (2) The use and purpose of the rubric; and (3) Perspectives on the rubric design. All three of these categories and their subthemes have been influenced by one amplifying factor: the experience level of the thesis supervisor, and their beliefs and practices relevant to the undergraduate thesis. The results will be discussed using the three major categories as a guiding framework.

\subsection{Perceptions of the Rubric and the Nature of the Thesis Course}

Through the interviews, we determined a number of interesting themes related to the nature of individual projects, and by extension the use of the rubric. For example, some supervisors seemed to think that the rubric was better suited to experimental rather than designoriented work, with one supervisor noting, for example, that "the components misaligned for a research article...for a design (project) I did not expect a journal paper and didn't have much issue with the structure.". Another supervisor felt the rubric was, in fact, not well suited for a design-oriented thesis, noting "when it's ( $a$ design thesis), there's not a lot of literature that you can use (when we) make something and then test it - oh that didn't work very well did it; what happened? Now we can respond and we're constantly iterating, so it doesn't really speak to an experimental methodology." Some supervisors indicated that the rubric was at odds, at least to some degree, with their own disciplinary traditions or understanding of the nature of research; for example "Ifeel like what I want to see is different from what engineering science wants to see... and that's partially (a) discipline issue...". Another supervisor noted that "sometimes, (our work) doesn't have a strong theoretical basis, but it has a computational or mathematical basis", demonstrating a need to expand the described conceptualization of "theoretical."

In some cases, the supervisors felt that the rubric miscategorized the purpose and aims of the undergraduate thesis. For example, in discussing some of the "Project Experience" dimensions of the rubric, one supervisor noted "I always interpret it in terms of what I expect for a fourthyear student. I've never had one that comes close to being publishable work. But for a fourth-year student they've learned an awful lot during the year so I kind of view that as very positive." Another supervisor noted "Work has contributed to scholarship in the field, made a measurable impact...well, probably not. It shouldn't be part of the grade." Another supervisor echoed this sentiment, noting "It's not an expectation on my part that they advance the field. If they just reinvent the wheel or take something one of my Masters students did and if nothing else corroborate that the work had been done properly I'm quite happy." Further, there was an acknowledgement that it might be up to the supervisor as to whether the student receives an opportunity to make an impact, with one supervisor noting "whether someone has a really highly impactful project really isn't up to the student because I kind of define it for them...". Interestingly, the components of the rubric that 
spoke to impact were added to help the supervisors distinguish the truly outstanding work from the good work that still meets the thesis requirements; these comments perhaps indicate a need to better justify rubric design decisions to the supervisors.

It was also suggested that the rubric may be interpreted differently, based on whether the project was studentinitiated or supervisor-initiated. This was especially evident when looking at the criteria around defining the goal of the work. One supervisor noted "(students) can go and describe very well what's been done, but it's hard for them to identify exactly what was the problem and what exactly is the goal...sometimes the supervisor is not very clear on what the goal is, to be honest", while another stated that "I'm always giving them high marks for (identifies goal), because I basically hand that one to them." It may be that this criterion needs to be broken down or further clarified, depending on how engaged the student was in actually crafting the goals of the research.

Finally, discussions about the rubric highlighted some concern about the focus on final product or process, and related to that, focus on project documentation or the ongoing research effort demonstrated across the semester. One supervisor noted "just looking at (the rubric) communicates that document design is more important than overall project experience...I take the liberty of being able to give them a grade based on what I think they deserve.", with another suggesting that "by the time we get to the final report, the final report is almost superfluous because I've been following them consistently and the final thing they do I already know how well they've been doing it and I've already been evaluating them." Another was firm in their conviction that the final grade should reflect the research experience: "I don't use the reports to assess my students' accomplishments. A student could do excellent work and be really bad at expressing what they've done in this report." Another supervisor tried to suggest a balanced approach, noting "I definitely read the documents and I want them to be good - I care about communication - but I want to keep the balance of what work they do, the effort they put into that, the science or engineering balanced against whether they write a great report and don't do anything." It seems that further clarification about the role of documentation vs project work would be useful in the process.

\subsection{The Use and Purpose of the Rubric}

The supervisors interviewed described what they used the rubric for, and how they went about using it, which resulted in some interesting contrasting themes. For example, some supervisors perceived the rubric as a way to communicate expectations to the student, for example, "I want them to adhere to the standard that their other peers are working towards". For some, it provided scaffolding for guiding the process; for example, one supervisor noted that "most of the use of rubrics has come well before that final evaluation. The useful part of it to me is to provide feedback earlier on..."

In some cases, supervisors indicated that it was a useful tool for communicating feedback, and some specifically noted, for example, that "I use this more as a feedback mechanism than a grading mechanism". However, it was clear that most of the supervisors were not using the rubric to help inform a final grade. For example, one supervisor noted "I more or less think of the mark that the student deserves from the start... and then the rubric is more, from my point of view, for feedback to the student, to tell them which of these little pieces they could have done better". Another supervisor noted "I don't know if (using the rubric) will result in increased accuracy than the usual more intuitive way of grading". Understanding how instructors perceive of "intuitive grading" would be an interesting area to explore further. Another supervisor noted that they "fill the rubric but it's frankly more of a post-hoc justification." Supervisors expressed some confusion about balancing the needs of the rubric and their own assessment, with one stating, "I can't just give them the mark that I know they deserve because the rubric is looking for certain things... in the end, my grade is largely based on what I know of them and the work they did." However, one supervisor specifically noted "What I found very helpful about the rubrics is that they formalized the whole process...the 1,2,3,4 process was very helpful in mapping the criteria to the actual mark the students got." This doesn't suggest that the supervisor is using the rubric results to craft the mark, but at least suggests congruence between their assessment and the rubric.

\subsection{Perspectives on the Rubric Design}

The interviews uncovered many concerns and reflections on the rubric design itself. Some supervisors struggled with the differentiation between levels, for example, "there's a lot of subtlety in some of (the criteria) in terms of the difference between a 3 and a 4."

Some supervisors noted that they felt components were missing. For example, one supervisor noted "particularly in my area...mathematics is quite central to any thesis the students are going to do...it might be helpful to explicitly highlight mathematical presentation and notation." Another supervisor noted "I would think that somewhere there should be...(an) ability to convey important scientific, technical or medical messages or something. I don't see it as a separate component." In discussing the role of goal identification, one supervisor suggested that "maybe scientific or technological/clinical hypothesis would be better than a purpose statement". With respect to results, one supervisor noted that "there's not a lot of emphasis on the actual findings - there's really a single line item here". Finally, one supervisor noted that "I don't expect a big comprehensive (literature) review but often 
they're the other extreme where they're too skimpy... "comprehensive" would imply a much bigger effort, but I think there's balance there that's often missing."

Some supervisors noted extraneous components, for example, "the second and third under literature review are kind of similar...in my mind I was focusing on the understanding of the field, understanding of the research...so I kind of treated them as the same.", while another supervisor noted "there's overlap between "outlines significance, identifies future work" and "significance" under (the introduction section)...I feel like they're two sides of the same point." Some supervisors felt that there were too many criteria, with one noting that "the high-level components are more useful than the very detailed things", and another sharing that "I think I can tell quite easily what something is worth without going through eighteen fine printed points." On supervisor shared their disagreement with some of the elements: "document length, format, structure - I have no idea what to put for that, I don't know what those requirements are and quite frankly, I disagree with a lot of that stuff." There was a sentiment from some of the supervisors that the final evaluation should be more holistic, for example, "I strongly oppose a fragmented rubric-based approach for the final evaluation...it me, it has to be on the world as a whole...was it convincing? Was it compelling?"

There were specific criteria, also reflected in our past work on interrater reliability, that caused confusion and were discussed in the interviews, for example, "Engages and explains results intelligently". One instructor interpreted this as "if something didn't work out, they should explain that something didn't work out". Another suggested that validation - "how do I know this is right" was the most critical component of explaining results intelligently. Another noted "I was looking at were they describing it in a way that you want another engineer to be able to read it and understand what the point is. So it has to be logical," while one supervisor questioned, "if (the experiment) did work, then what? There's not much you can do to explain your results intelligently..." Finally, one supervisor noted that "(The term) "engages with" is kind of strange. You mean has a captivating writing style and...provides context for scientific discussion?".

When it came to discussions on how we might support the use of the rubrics, there was some interest in seeing specific examples, but less interest in group benchmarking, although one supervisor did note "I think it would be neat for me to talk to another professor who is marking a report ... because I do feel like a bit of a lone ranger the day I decide to mark it...". However, another supervisor noted that he only had a "passing curiosity...I don't think I'd want to engage directly because I feel like there's almost a slightly confrontational element to that... what if you were the supervisor who's doing a bad job?" Some supervisors expressed an opinion that normative based assessment was important to them, with one noting that "giving more information on averages or target averages might help to (normalize grading) in a more succinct way". Finally, some supervisors noted the challenge in completing the worded feedback sections, for example, "I find these blank boxes a little intimidating...I don't know whether some leading questions might help in that regard." Overall, supervisors tended to be interested in the possibility of seeing graded examples at different levels of competency, that they could access as they needed.

\section{CONCLUSIONS AND FUTURE WORK}

Based on the results described in this paper, there are a number of issues related to the use, purpose and design of the rubric that we need to consider as we move forward in the evolution and use of the rubrics. The results of this study will be used to create an online survey for a larger group of supervisors, which will further explore the use of the rubrics in the entire supervisor population, and some of the themes raised by supervisors; for example, whether there is a need to further articulate a separation between documentation and project work; whether there is a need to provide customization of the rubric depending on the project type, and how to best clarify some of the confusing criteria. It will also be interesting to collect a more widespread view on how the rubric is being used - for example, to help with summative assessment, to guide the thesis process, to provide feedback to students or communicate expectations, and to determine what the implications are of a diverse set of uses. The survey will also allow us to better determine whether the rubric criteria are aligning with what the supervisors feel is important as part of an undergraduate thesis.

\section{Acknowledgements}

We would like to acknowledge the Faculty members who graciously participated in the interviews, and the students who have volunteered their thesis work and assessment for this project. The work reported in this paper has been supported by the Faculty of Applied Science and Engineering's Educational Instructional Innovation Program Grant.

\section{References}

[1] Alan Chong and Lisa Romkey, "Adapting existing assessment tools for use in assessing engineering graduate attributes", in Proc. CEEA Canadian Engineering Education Conf., CEEA12, Ron Britton (ed.) (Winnipeg, MB; 17-20 June 2012), 16 pp., 2012.

[2] Alan Chong and Lisa Romkey, "Pedagogical innovations to support large scale undergraduate independent research projects", in Proc. CEEA Canadian Engineering Education Association Conf., CEEA14, Sean Maw (ed.) (Canmore, AB; 8-11 June 2014), 1 pp., 2014. 
[3] Alan Chong and Lisa Romkey, "Testing Inter-Rater Reliability in Rubrics for Large Scale Undergraduate Independent Projects", in Proc. CEEA Canadian Engineering Education Association Conf., CEEA16, Clifton Johnston (ed.) (Halifax, NS; 19-22 June 2016), 9 pp., 2016.

[4] Nikita Dawe, Lisa Romkey, Susan McCahan, and Gayle Lesmond, "User testing with assessors to develop universal rubric rows for assessing engineering design", in Proc. ASEE Annual Conference \& Exposition, (New Orleans, LA; 26-29 June 2016), 14 pp., 2016.

[5] Carol Grbich, "Qualitative Data Analysis: An Introduction", London, UK: Sage Publications, 2007.

[6] Gayle Lesmond, Nikita Dawe, Susan McCahan, and Lisa Romkey, "Update on the Development of Analytic Rubrics for Competency Assessment (DARCA)", in Proc. CEEA
Canadian Engineering Education Association Conf., CEEA16, Clifton Johnston (ed.) (Halifax, NS; 19-22 June 2016), 6 pp., 2016.

[7] Michael Quinn Patton, "Qualitative Research \& Evaluation Methods: Integrating Theory and Practice", Thousand Oaks, Calif: Sage Publications, 2014 (fourth edition).

[8] Lisa Romkey, Alan Chong, and Lobna El Gammal, "Using student focus groups to support the validation of rubrics for large scale undergraduate independent research projects", in Proc. CEEA Canadian Engineering Education Association Conf., CEEA15, Thomas Doyle (ed.) (Hamilton, ON; 31 May - 3 June 2015), 8 pp., 2015. 


\section{APPENDIX A: RUBRIC AND RUBRIC GUIDE}

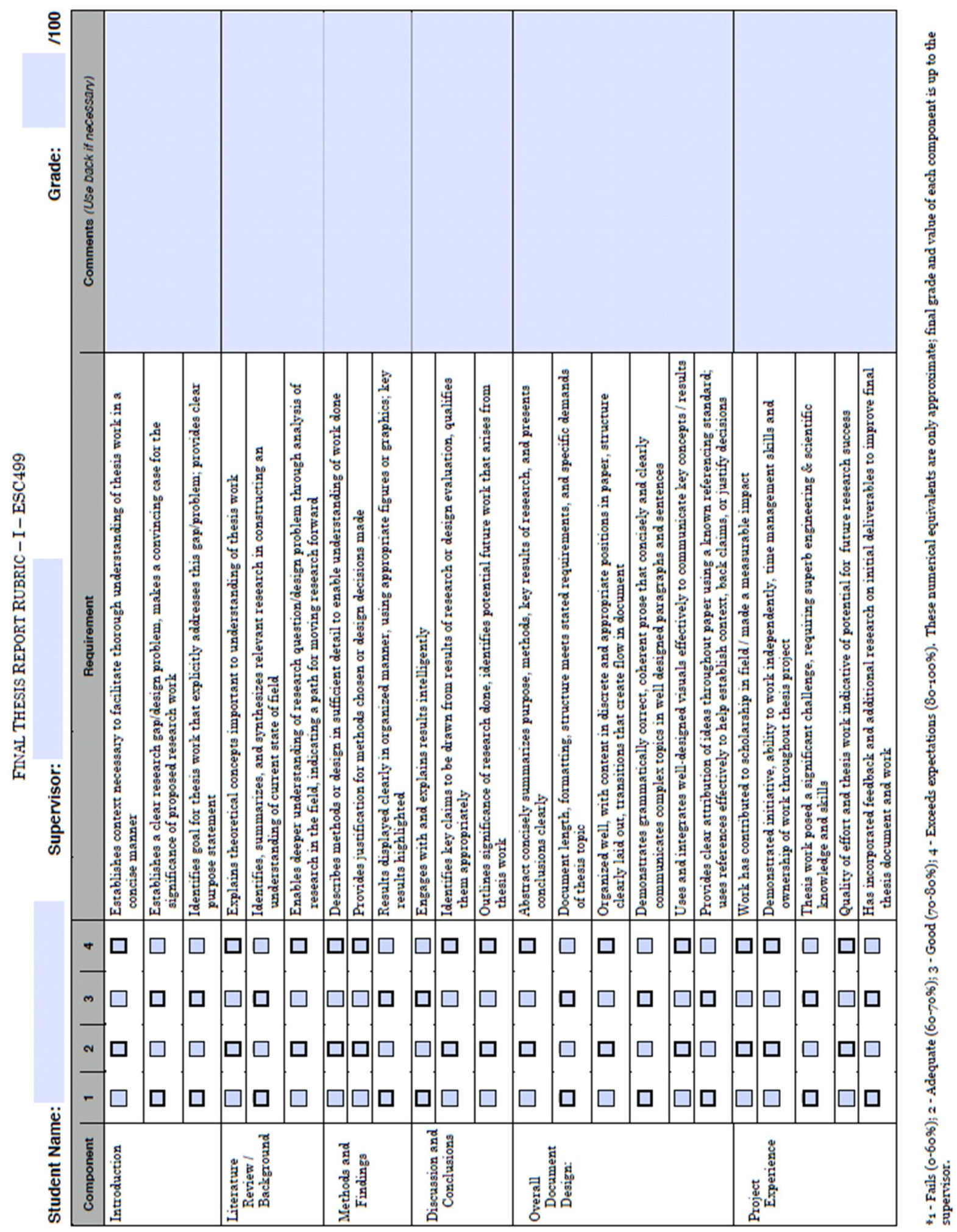




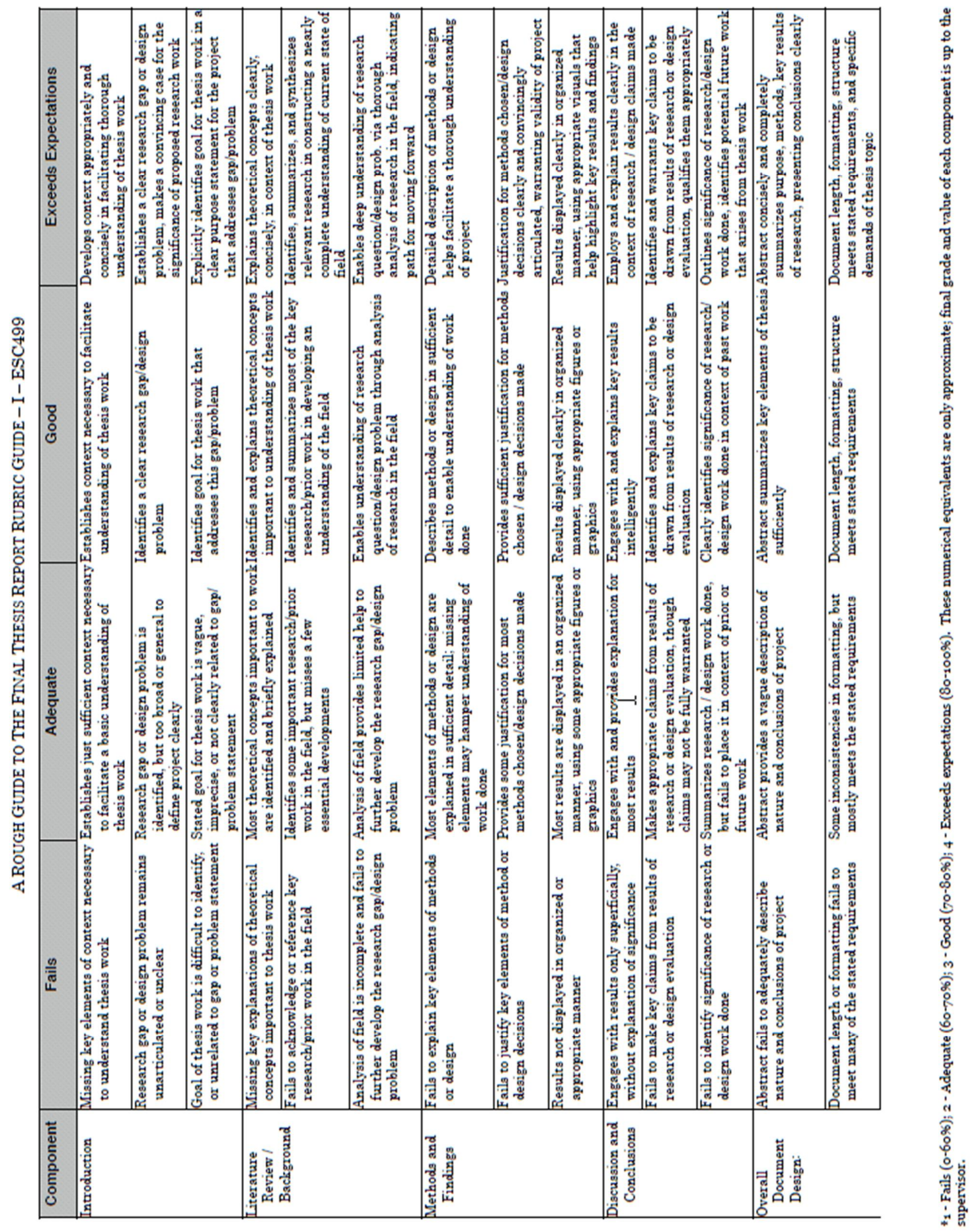




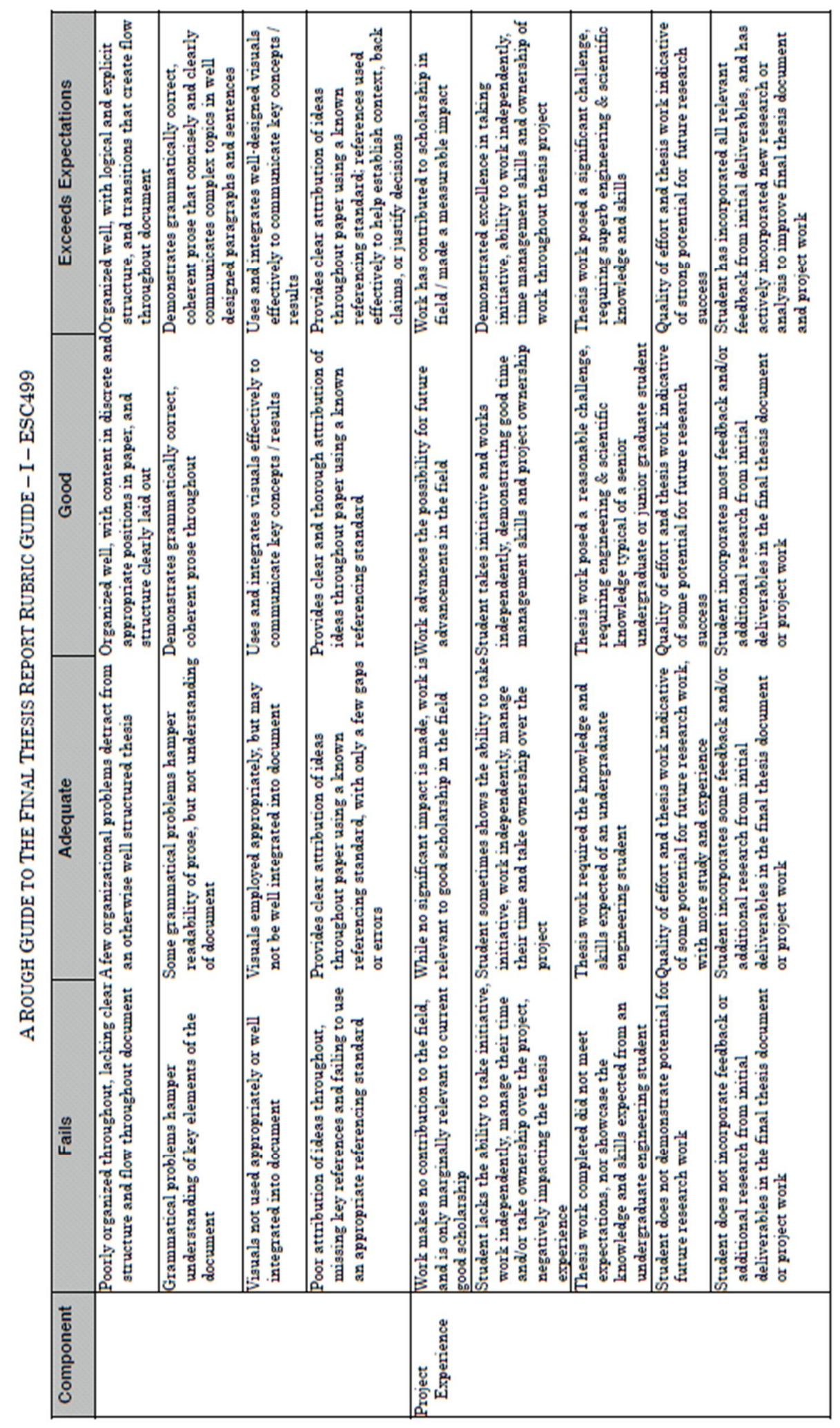

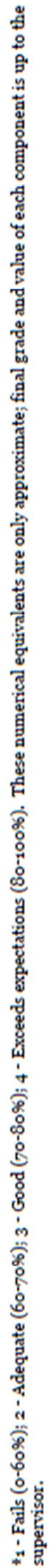

